\title{
Non-paraxial Talbot effect in one-dimensional gratings with period comparable to the wavelength
}

\section{Efecto Talbot no paraxial en una red unidimensional de periodo comparable a la longitud de onda}

\author{
E. Arrieta ${ }^{1}$, N. Bolognini ${ }^{2}$, C. O. Torres ${ }^{3}$ \\ ${ }^{1}$ PhD student in Physics SUE-Caribe. Grupo de Óptica e Informática (LOI), \\ Universidad Popular del Cesar, Valledupar- Colombia. \\ ${ }^{2}$ Centro de Investigaciones Ópticas, CIOp (CONICET La Plata-CIC),C.C. 3,1897, M. B. Gonnet, Argentina. \\ ${ }^{3}$ Grupo de Óptica e Informática (LOI), Universidad Popular del Cesar, Valledupar-Colombia. \\ 1E-mail: earrietajimenez@yahoo.es
}

Received: 04/11/2016 Accepted:29/05/2017

DOI: $10.7149 /$ OPA.50.2.49019

\begin{abstract}
The Talbot effect is a diffractive phenomenon which was found since its inception in the Gaussian optics, but when the period of the object is comparable with the wavelength is considered entering its non-paraxial regime. There, it has attracted interest for its applications related to the design of objects and their restorative effects posing in damaged gratings on this scale. In this contribution a study of this phenomenon at level non-paraxial (metaxial) is presented by using scalar diffraction theory in plane waves representation for periodic transversal fields. To this end, a one-dimensional amplitude grating of period $p$ is used which is illuminated by a monochromatic plane wave of wavelength $\lambda$ where p ranges between $1,5 \lambda$ and $4,2 \lambda$.
\end{abstract}

Key words: Talbot effect, amplitude grating, non paraxial optics.

\section{RESUMEN}

El efecto Talbot es un fenómeno difractivo ubicado desde su origen en la óptica Gaussiana, pero cuando la longitud de onda es comparable con el periodo del objeto se considera que este efecto entra en su régimen no paraxial, el cual nuevamente ha despertado el interés por su volumen de aplicación relacionado con el diseño de objetos y sus efectos curativo que presenta en redes averiadas a esta escala En esta contribución presentamos un estudio de este fenómeno a nivel no paraxial (metaxial),usando la teoría escalar de difracción en representación de ondas planas para campos periódicos transversal. Para ello se utiliza como objeto periódico una red de amplitud unidimensional de periodo $\mathrm{p}$, iluminada con una onda plana monocromática de amplitud unitaria y longitud de onda $\lambda$, donde p se encuentra entre $1,5 \lambda$ y $4,2 \lambda$.

Palabras clave: Efecto Talbot, red de amplitud, óptica no-paraxial.

\section{REFERENCES}

[1] Smolyaninov I. and Davis C. "Apparent super-resolution in near-field optical imaging of periodic gratings", Opt. Lett, 23(17): 1346-1347 (1998).

https://doi.org/10.1364/OL.23.001346

[2] Yi Hua, Suh Jae Yong, Zhou Wei, Huntington Mark D and Odom Teri W, "Talbot effect beyond the paraxial limit at optical frequencies", Opt. Express, 20(13):14284-14291 (2012). https://doi.org/10.1364/OE.20.014284

[3] Flores-Arias M. T., C. Bao, Pérez .M. V and Fernández-Pousa .C. R, "Fractional Talbot effect in a Selfoc gradient-index lens", Opt Lett, 27(23): 2064-2066 (2002).

https://doi.org/10.1364/OL.27.002064 
[4] Guérineun Nicolas, Di Mambro Emmanuel and Primot Jérome,"Talbot experiment re- examined: study of the chromatic regime and application to spectrometry", Opt. Express,11(24):3310-3318 (2003). https://oi.org/10.1364/OE.11.003310

[5] Ring James D, Lindberg Jari, Howls Christopher J. and Dennis Mark R, "Aberration-like cusped focusing in the post-paraxial Talbot effect", arXiv: 1204.5994 v1[physics optics] (2012).

[6] Dennis Mark R, Zheludev Nikolay I, and Garcia de Abajo F Javier, "The plasmon Talbot effect", Opt. Express, 15(15):9692-9700 (2007). https://doi.org/10.1364/OE.15.009692

[7] Zhang Weiwei, Zhao Chenlong, Wang Jiayuan, and Zhang Jiasen "An experimental study of the plasmonic Talbot effect”, Opt. Express, 17(22):19757-19762 (2009). https://doi.org/10.1364/OE.17.019757

[8] Cherukulappurath Suhir, Heinis Dominique, Cesario Jean, van Hulst Niek F, Enoch Stefan, and Quidant Romain, "Local Observation of plasmon focusing in Talbot carpets", Opt. Express, 17(26):2377223784 (2009). https://doi.org/10.1364/OE.17.023772

[9] Zhang Young, Wen Jianming, Zhu S. N, and Xiao Min, "Nonlinear Talbot Effect", Phys. Rev. Lett, 104(18):3901 -3904 (2010). https://doi.org/10.1103/PhysRevLett.104.183901

[10] Wei Gong X., Lu Lei L., Guo Cheng S., "Generation of optical vortex array based on the fractional Talbot effect", Opt. Commun., 282: 2665-2669 (2009). https://doi.org/10.1016/j.optcom.2009.04.001

[11] Forte Gustavo, Lencina Alberto, Tebaldi Myrian, Bolognini Nestor, "Self-imaging by a volume grating" Opt. Commun., 284:2494-2499 (2011). https://doi.org/10.1016/j.optcom.2011.01.044

[12] Forte Gustavo, Lencina Alberto, Tebaldi Myrian, Bolognini Nestor, "Talbot effect by a photorefractive volume phase grating", Appl. Opt, 51(4):479-485 (2012). https://doi.org/10.1364/AO.51.000479

[13] Martínez León L, Araiza E M, Javidi B, Andrés P, Climent V, Lancis J, and Tajahuerce E, “ Single-shot digital holography by use of the fractional Talbot effect”, Opt. Express, 17(15):12900-12908 (2009). https://doi.org/10.1364/OE.17.012900

[14] Bozhevolnyi S I, Xiao M, and Keller 0, "External-reflection near-field optical microscope crosspolarized detection”, Appl. Opt, 33(5):876-880 (1994). https://doi.org/10.1364/AO.33.000876

[15] Lord Rayleigh,"On copying diffraction-gratings and on some phenomena connected therewith" Philos. Mag. 11(67):196-205 (1880). https://doi.org/10.1080/14786448108626995

[16] Saastamoinen T., Tervo J., Vahimaa P., and Turunen J.," Exact self-imaging of transversely periodic fields “, J. Opt. Soc. Am.A, 21(8):1424-1429 (2004). https://doi.org/10.1364/JOSAA.21.001424

[17] Teng Shuyun, Chen Xiaoyi, Zhou Tongjun, and Cheng Chuanfu," Quasi-Talbot effect of a grating in the deep Fresnel diffraction region", J. Opt. Soc. Am. A, 24(6):1656-1665 (2007). https://doi.org/10.1364/JOSAA.24.001656

[18] Teng Shuyun, Zhang Ningyu, Dong Qingrui, and Cheng Chuanfu, "Diffraction of a one-Dimensional phase grating in the deep Fresnel field", J. Opt. Soc. Am. A, 24(11):3636-3643 (2007). https://doi.org/10.1364/JOSAA.24.003636

[19] Teng Shuyun, Tan Yugui, and Cheng Chuanfu,"Quasi-Talbot effect of the high-density grating in near field”,J. Opt. Soc. Am. A, 25(12):2945-2951 (2008). https://doi.org/10.1364/JOSAA.25.002945

[20] Arrieta. E.S, Torres, C.O, and Bolognini. N, "Metaxial Talbot effect in free space", Proc. SPIE, Vol. 9660:1-4 (2015).

\section{Introduction}

The Talbot effect is displayed when a grating is illuminated with a plane wave and consists in the presence of self-images of the grating at determined planes. These images are generated by propagation in free 
space of the diffracted optical field. When the location of these planes is related to the so-called Talbot distance, the paraxial regime takes place. In the (non-paraxial) metaxial regime at optical frequencies, the position of the self-images does not correspond to the Talbot distance and can be used to observe abnormalities in periodic (or quasi-periodic) objects that are perfect in the paraxial regime, i.e., achieving "super-resolution" on this scale [1,2]. The paraxial Talbot effect among other applications is used to increase or decrease the spatial frequency, in integrated optics for optical coupling, in microscopy techniques and image restoration. It has been studied at different scales and in different propagation media. Thus, it has been shown in waveguides, in designing the components of integrated optics and multiplexed applications, and also in an inhomogeneous medium with gradient index micro-lenses, giving an idea of this effect at micrometric scale [3]. In addition, it has been investigated the chromatic regime and its application to spectrometry [4], aberrations of the self-images in the paraxial regime [5], in the study of plasmonic waves [6-8] and nonlinear photonic crystals [9], generation of optical vortices [10], and more recently with volume grating in photorefractive crystals [11,12] and digital holography [13].

On the other hand, the phenomenon has been studied with high density gratings in the deep region of Fresnel diffraction, which shows that there are self-images, longitudinally non-periodic, positioned by an empirical relationship and is called quasi-Talbot effect [17-19]. Later, the relationship was established from the scalar diffraction theory [20]. In this contribution the study of non paraxial Talbot effect is deepened by an analysis of propagating wave modes that contribute most to self-images formation for gratings with periods between $2 \lambda$ and $4,2 \lambda$.

\section{Non-paraxial Talbot effect}

Let us consider a non-limited amplitude grating as a one-dimensional periodic distribution. The scalar field behind the grating of period $p$ positioned at the origin $(z=0)$, illuminated by a unitary amplitude plane wave normally incident can be written:

$$
E(x, z)=\sum_{n} C_{n} \exp (2 \pi i n x / p) \exp \left(i k_{z}^{n} z\right), \quad k_{z}^{n}=\left((2 \pi / \lambda)^{2}-(2 \pi n / p)^{2}\right)^{1 / 2}
$$

Where $C_{n}$ is a Fourier coefficient. Let us consider that $p>>\lambda$, then $k_{n} z \approx 2 \pi z / \lambda-2 \pi n^{2}\left(\lambda z / 2 p^{2}\right)$. Taking into account that the square of an integer $n$ is an integer, at $\mathrm{z}=Z_{T}=2 p^{2} / \lambda$ (Talbot distance) all the Fourier components of the optical field exactly have the same relative phase differences as they have at the plane $z=0$. Thus, the grating field distribution replicates itself. These self-images are observed at distances that are multiples of the Talbot distance, that is $z=n Z_{T}$. More complex field patterns occur at fractional Talbot distance values. These patterns are localized to where $z=(q / \mathrm{s}) Z_{T}$ with $q$ and $s$ are integers. In the case of fractional Talbot planes, many Fourier components of the field (but not all) have the same relative phase difference and the field pattern is similar to the field distribution at $z=0$, but not reproduced exactly $[1,20]$.

The condition to achieve exact self-images requires that all plane waves are in phase at the same distance. If the center frequency $\left(0,{k_{z}}^{0}\right)$ is chosen as the reference, this condition can be written as $[1,2,20]$ :

$$
\exp \left[\mathrm{ik}_{z}^{0} \mathrm{z}_{R}\right]=\exp \left[\mathrm{ik}_{z}^{n} \mathrm{z}_{R}\right]
$$

By using the first two frequencies it is:

$$
\mathrm{z}_{R}=\lambda /\left(1-\sqrt{1-(\lambda / \mathrm{p})^{2}}\right)
$$

Eq. (3) represents the Rayleigh distance which is an approximation of the metaxial Talbot regime [2, 15]. Using the same condition (2) for self-images and taking an arbitrary component propagating mode $m$ as reference that participates in the formation of self-image, the general expression [20] is obtained:

$$
\mathrm{z}_{m n}=\lambda C_{m n} /\left(\sqrt{1-(\lambda m / \mathrm{p})^{2}}-\sqrt{1-(\lambda n / \mathrm{p})^{2}}\right)
$$


Which determines the location of the self-images in near field, and the corresponding $\pi$ radians shifted self-image results:

$$
\mathrm{z}_{m n}=\lambda\left(s-\frac{1}{2}\right) C_{m n} /\left(\sqrt{1-(\lambda m / \mathrm{p})^{2}}-\sqrt{1-(\lambda n / \mathrm{p})^{2}}\right)
$$

where $C_{n m}, m, n$ and s are integers, being $m<n$.

\section{Results and analysis}

In this one-dimensional case, consider a periodic object with $\mathrm{p}=1,936 \mu \mathrm{m}$ illuminated with a plane wave of unit amplitude and wavelength $\lambda=633 \mathrm{~nm}$ (i.e. $p \approx 3 \lambda$ ) as in references $[1,14,20]$. The maximum number of propagating waves, according to the values of $p$ and $\lambda$ is $n=3$. By considering Eq. (1) and neglecting the evanescent waves, the field distribution is:

$$
E_{1}(x, z)=\sum_{n=-3}^{3} C_{n} \exp (2 \pi i n x / p) \exp \left[2 \pi i\left(\frac{1}{\lambda^{2}}-\frac{n^{2}}{p^{2}}\right)^{1 / 2} z\right]
$$

Be $w$ the width of each slit grating. By assuming that $p<<w$, in this case, the coefficients $C_{n}$ meet the condition $C_{1}=C_{2}=C_{3}$. The respective intensity patterns are displayed at different z-positions with the help of the program Mathematica. We show in the following figures the object as in the work of ref. [20], and some relevant self-images.

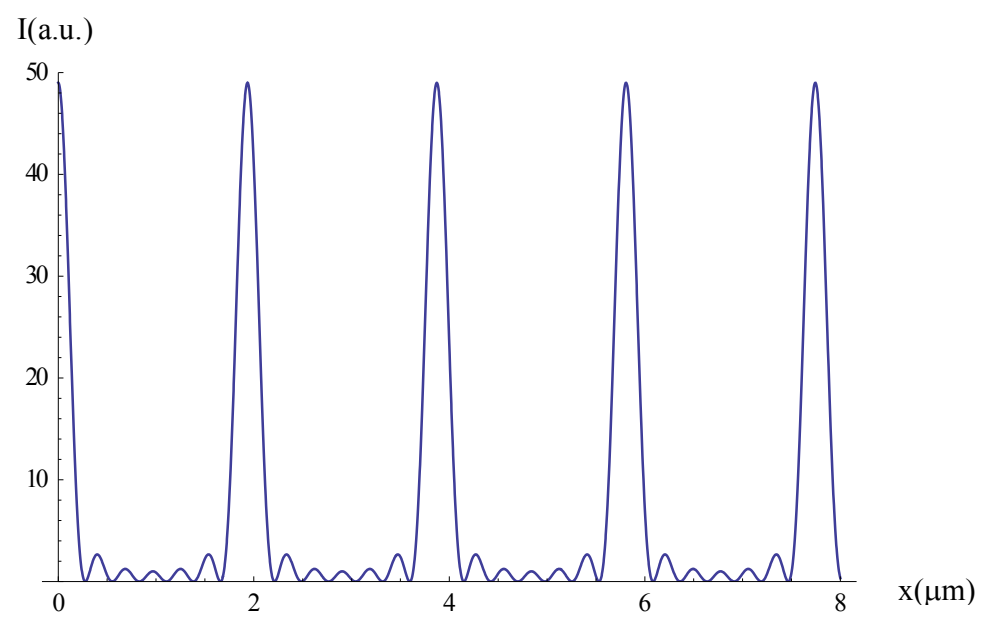

Fig. 1. Object at $z=0 \mu \mathrm{m}$.

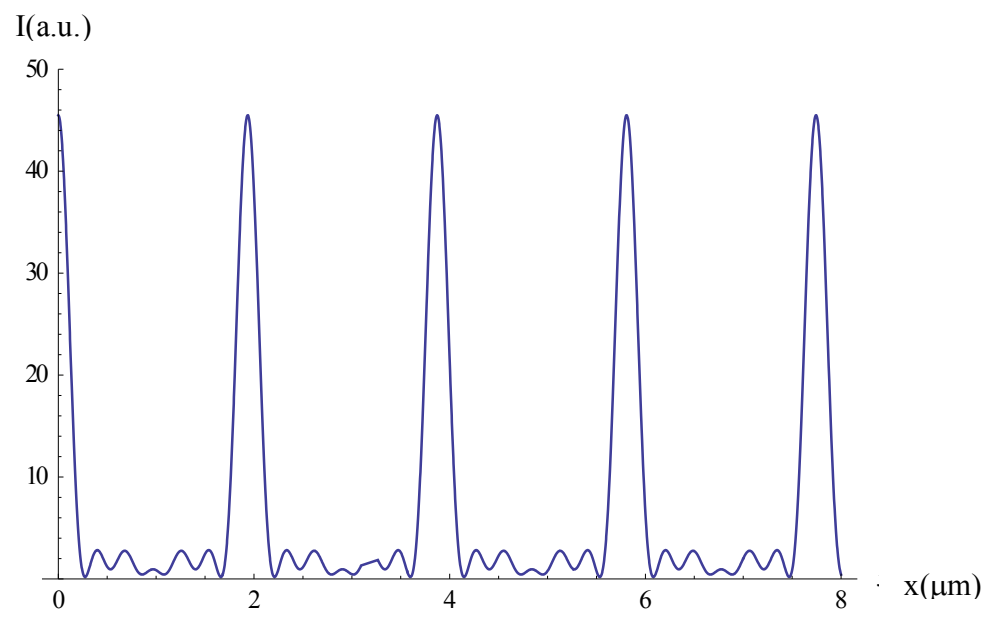

Fig. 2. Self-image at $z=10,20 \mu \mathrm{m}$ [20]. 


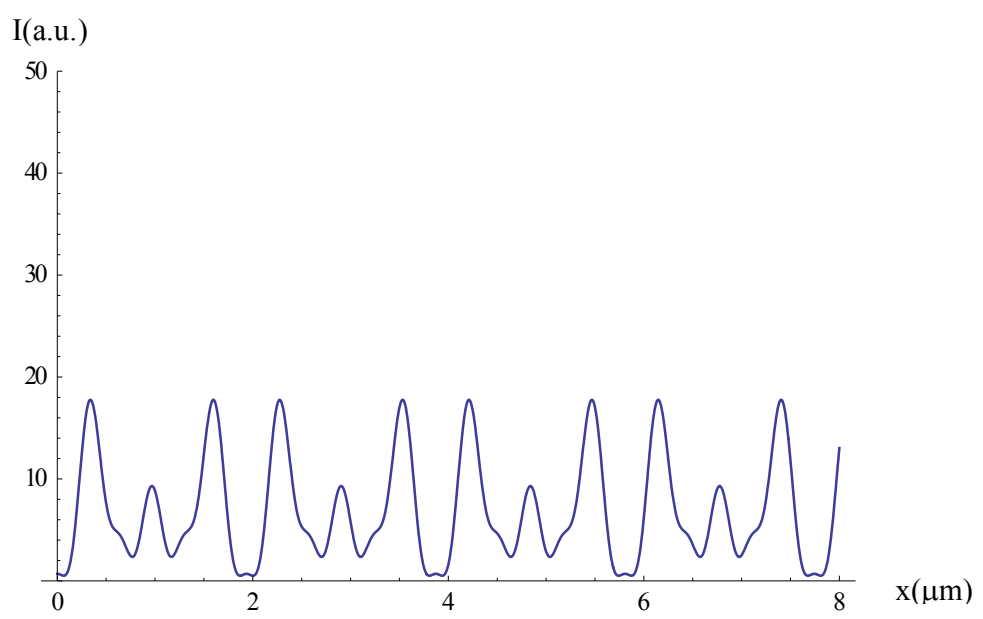

Fig .3. Quasi self-image at $Z_{R}=11,52 \mu \mathrm{m}$.

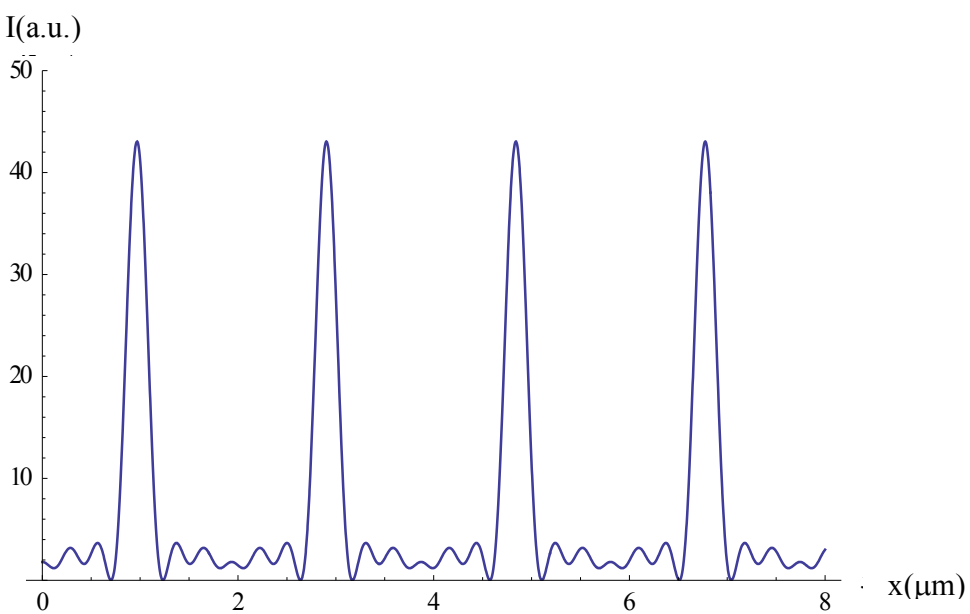

Fig. 4. Self-image at $\mathrm{z}=15,26 \mu \mathrm{m}[20]$.

By observing these graphics, it can be inferred that at the Rayleigh distance no self-image is formed as shown in Fig. 3 and that the true self-images around it are formed at 10, $20 \mu \mathrm{m}$ and 15, $26 \mu \mathrm{m}$ as we observe in Fig. 2 and Fig. 4, respectively. This indicates that we are employing a grating with period comparable to the wavelength used. Finally, the values of $Z_{m n}$ by using Eq. (4) and Eq. (5) are obtained in Fig. 2 with $C_{12}=3, C_{13}=12, C_{23}=9$ and in Fig. 4 with s $=2, C_{12}=3, C_{13}=12, C_{23}=9$, respectively.

When we consider the self-image formation by including the first two evanescent waves, that is, assuming $n=4$ in Eq. (1), we note that self-images are the same as previously found (for $n=3$ ). It indicates that the treatment of metaxial Talbot effect in free space only encompasses propagating waves. Then, it implies that is an optical far field phenomenon in such gratings with period in the range of wavelengths, and not as in ref.[14] the authors had experimentally proposed by using near-field microscopy, and was noted and corrected in ref. [1] .

Hereinafter, we consider the formation of self-images evaluating its location using Eq. (4) and (5) for different relationships between the period and the wavelength. The intensity of the self or quasi-self images are obtained from the optical field presented in Eq. (6) at different axial positions by operating the program Mathematica and using it's manipulate command. In all cases a one dimensional amplitude gratings is employed.

\section{3.a. Grating with $p=1 \mu \mathrm{m}, \lambda=0,616 \mu \mathrm{m}(\mathrm{p}=1,623 \lambda)$.}

In this case, the propagating waves in the self image formation are for $n=-1,0$, 1 . Therefore, the nonparaxial expression reduces to the Rayleigh equation. In Table 1, the position interval, the self-image best position, kind of image, contrast $\mathrm{C}\left(C_{\text {image }}=\left(\mathrm{I}_{\max }-\mathrm{I}_{\min }\right) /\left(\mathrm{I}_{\max }+\mathrm{I}_{\min }\right)\right.$ and $\mathrm{C}(\%)=\mathrm{C}_{\text {imagex }} 100 / \mathrm{C}_{\text {object }}$ with I: 
intensity) in percentage (\%) and the $C_{m n}$ values are displayed. The lowest contrast to observe self-images is from $60 \%$.

TABLE 1. Location and image quality obtained with scalar diffraction theory, for a $1 \mathrm{D}$ grating with $\mathrm{p}=1.623 \lambda$ and $\lambda=0.616 \mu \mathrm{m}$.

\begin{tabular}{|c|c|c|c|c|}
\hline $\begin{array}{c}\text { Position } \\
\text { Interval }(\mu \mathrm{m})\end{array}$ & $\begin{array}{l}\text { Position } \\
(\mu \mathrm{m})\end{array}$ & Image type & $\mathrm{C}(\%)$ & $C_{m n}$ and $s$ \\
\hline $0,72-0,74$ & 0,73 & Fractional & 63,6 & $C_{01}=1, C_{01} / 4$ \\
\hline $1,10-1,80$ & 1,45 & Integer $\pi$-shifted & 100 & $C_{01}=1, s=1$ \\
\hline $2,15-2,20$ & 2,17 & Fractional & 63,6 & $C_{01}=13, C_{01} / 4$ \\
\hline $2,60-3,20$ & 2,90 & Integer & 100 & $C_{01}=1$ \\
\hline $3.60-3,65$ & 3,63 & Fractional & 63,6 & $C_{01}=15, C_{01} / 4$ \\
\hline $3,80-4,50$ & 4,30 & Integer $\pi$-shifted & 100 & $C_{01}=1, s=2$ \\
\hline $5,05-5,10$ & 5,07 & Fractional & 63,6 & $C_{01}=1,7 C_{01} / 4$ \\
\hline $5,30-5,90$ & 5,80 & Integer & 100 & $C_{01}=2$ \\
\hline $6,50-6,55$ & 6,53 & Fractional & 63,6 & $C_{01}=1,9 C_{01} / 4$ \\
\hline $6,90-7,50$ & 7,20 & Integer $\pi$-shifted & 100 & $C_{01}=1, s=3$ \\
\hline $7,95-8,00$ & 7,98 & Fractional & 63,6 & $C_{01}=1,11 C_{01} / 4$ \\
\hline $8,20-8,90$ & 8,70 & Integer & 100 & $C_{01}=3$ \\
\hline $9,42-9,45$ & 9,43 & Fractional & 63,6 & $C_{01}=1,13 C_{01} / 4$ \\
\hline $9,80-10,40$ & 10,20 & Integer $\pi$-shifted & 100 & $C_{01}=1 \cdot s=4$ \\
\hline $10,86-10,90$ & 10,88 & Fractional & 63,6 & $C_{01}=1,15 C_{01} / 4$ \\
\hline $11,20-11,90$ & 11,60 & Integer & 100 & $C_{01}=4$ \\
\hline $12,31-12,36$ & 12,33 & Fractional & 63,6 & $C_{01}=1,17 C_{01} / 4$ \\
\hline $12,70-13,30$ & 13,10 & Integer $\pi$-shifted & 100 & $C_{01}=1, s=5$ \\
\hline $13,76-13,80$ & 13,78 & Fractional & 63,6 & $C_{01}=1,19 C_{01} / 4$ \\
\hline $14,10-14,80$ & 14,50 & Integer & 100 & $C_{01}=5$ \\
\hline $15,21-15,26$ & 15,23 & Fractional & 63,6 & $C_{01}=1,21 C_{01} / 4$ \\
\hline $15,60-16,20$ & 15,95 & Integer $\pi$-shifted & 100 & $C_{01}=1, s=6$ \\
\hline $16,67-16,70$ & 16,68 & Fractional & 63,6 & $C_{01}=1,23 C_{01} / 4$ \\
\hline $17,00-17,70$ & 17,40 & Integer & 100 & $C_{01}=6$ \\
\hline $18,12-18,15$ & 18,13 & Fractional & 63,6 & $C_{01}=1,25 C_{01} / 4$ \\
\hline $18,60-19,20$ & 18,90 & Integer $\pi$-shifted & 100 & $C_{01}=1, s=7$ \\
\hline $19,56-19,60$ & 19,58 & Fraccional & 63,6 & $C_{01}=1,27 C_{01} / 4$ \\
\hline $19,90-20,50$ & 20,30 & Integer & 100 & $C_{01}=7$ \\
\hline
\end{tabular}

The results of Table 1 imply that the grating shows an axial periodicity for the self-images similar to the classical case, that is when the grating period is much larger than the wavelength.

3.b. Grating with $p=1,226 \mu \mathrm{m}, \lambda=0,633 \mu \mathrm{m}(p=2 \lambda)$.

In this case, the propagating waves in the self image formation are for $n=-2-1,0,1,2$. In Table 2 , the position interval, the self-image best position, kind of image, contrast C in percentage (\%) and the $C_{m n}$ values are displayed. 
TABLE 2. Location and image quality obtained with scalar diffraction theory, for a $1 \mathrm{D}$ grating with $\mathrm{p}=2 \lambda$ and $\lambda=0.616 \mu \mathrm{m}$.

\begin{tabular}{|c|c|c|c|c|}
\hline \hline $\begin{array}{c}\text { Position } \\
\text { Interval }(\mu \mathrm{m})\end{array}$ & $\begin{array}{c}\text { Position } \\
(\mu \mathrm{m})\end{array}$ & $\begin{array}{c}\text { Image } \\
\text { type }\end{array}$ & $\mathrm{C}(\%)$ & $C_{m n}$ \\
\hline \hline $1,42-1,46$ & 1,44 & Integer & 80 & $C_{12}=2$ \\
$3,64-3,70$ & 3,68 & Integer & 85 & $C_{02}=6, C_{12}=5$ \\
$4,36-4,46$ & 4,40 & Integer & 95 & $C_{12}=6, C_{02}=7, C_{01} \approx 1$ \\
$5,04-5,12$ & 5,08 & Integer & 95 & $C_{12}=7, C_{02}=8, C_{01} \approx 1$ \\
$5,82-5,86$ & 5,84 & Integer & 80 & $C_{02} \approx 9, C_{12}=8$ \\
$8,04-8,08$ & 8,06 & Integer & 75 & $C_{02} \approx 13, C_{12}=11$ \\
$8,74-8,82$ & 8,78 & Integer & 95 & $C_{02}=14, C_{12}=12$ \\
$9,38-9,58$ & 9,48 & Integer & 100 & $C_{01}=2, C_{02}=15, C_{12}=13$ \\
$10,18-10,24$ & 10,22 & Integer & 85 & $C_{02}=16, C_{12}=14$ \\
$13,14-13,20$ & 13,16 & Integer & 80 & $C_{12}=18, C_{02} \approx 21, C_{01} \approx 3$ \\
$13,84-13,90$ & 13,88 & Integer & 90 & $C_{12}=19, C_{02} \approx 22, C_{01} \approx 3$ \\
\hline \hline
\end{tabular}

\section{3.c. Grating with $p=2,595 \mu \mathrm{m}, \lambda=0,633 \mu \mathrm{m}(p=4,1 \lambda)$.}

In this case, the propagating waves in the self- image formation are for $n=-4-3-2-1,0,1,2,3,4$. In Table 3 , the position interval, the self-image best position, kind of image, contrast $\mathrm{C}$ in percentage (\%) and the $C_{m n}$ values are displayed.

TABLE 3. Location and image quality obtained with scalar diffraction theory, for a $1 \mathrm{D}$ grating with $\mathrm{p}=4,1 \lambda$ and $\lambda=0.616 \mu \mathrm{m}$.

\begin{tabular}{|c|c|c|c|c|}
\hline $\begin{array}{c}\text { Position } \\
\text { Interval }(\mu \mathrm{m})\end{array}$ & $\begin{array}{l}\text { Position } \\
\text { ( } \mu \mathrm{m})\end{array}$ & Image type & C (\%) & $C_{m n}$ \\
\hline $6,78-6,86$ & 6,82 & Integer & 66,3 & $\begin{array}{c}C_{02} \approx 1, C_{03} \approx 3, C_{04} \approx 8, C_{12}=1, C_{13}=3, \\
C_{23}=2, C_{14}=8, C_{24}=7, C_{34}=5\end{array}$ \\
\hline $8,82-8,98$ & 8,90 & $\begin{array}{l}\text { Integer } \pi- \\
\text { shifted }\end{array}$ & 82,5 & $\begin{array}{c}C_{02} \approx 2, C_{03} \approx 4, C_{04} \approx 11, C_{12} \approx 1, C_{13}=4, C_{23} \\
\approx 3, C_{14}=10, C_{24}=9, C_{34} \approx 6\end{array}$ \\
\hline $10,58-10,66$ & 10,64 & $\begin{array}{l}\text { Integer } \pi- \\
\text { shifted }\end{array}$ & 68,8 & $\begin{array}{c}C_{02} \approx 2, C_{03} \approx 5, C_{04}=13, C_{12} \approx 2, C_{13} \approx 5, C_{23} \\
\approx 3, C_{14} \approx 12, C_{24} \approx 11, C_{34} \approx 8\end{array}$ \\
\hline $13,76-13,78$ & 13,74 & Integer & 62,5 & $\begin{array}{c}C_{02} \approx 3, C_{03} \approx 7, C_{04}=17, C_{12} \approx 2, C_{13} \approx 6, C_{23} \\
\approx 4, C_{14} \approx 16, C_{24}=14, C_{3} \approx 10\end{array}$ \\
\hline $14,74-14,84$ & 14,80 & Integer & 65 & $\begin{array}{c}C_{02}=3, C_{03} \approx 7, C_{04} \approx 18, C_{12} \approx 2, C_{13} \approx 7, C_{23} \\
\approx 5, C_{14} \approx 17, C_{24}=15, C_{34} \approx 11\end{array}$ \\
\hline $19,46-19,66$ & 19,61 & Integer & 88,8 & $\begin{array}{c}C_{02} \approx 4, C_{03} \approx 10, C_{04} \approx 24, C_{12}=3, C_{13} \approx 9, \\
C_{23}=6, C_{14}=23, C_{24}=20, C_{34}=14\end{array}$ \\
\hline $20,36-20,54$ & 0,48 & Integer & 63 & $\begin{array}{l}C_{01} \approx 1, C_{02}=4, C_{03} \approx 10, C_{04}=25, C_{12}=3, \\
C_{13} \approx 9, C_{23} \approx 6, C_{14}=24, C_{24} \approx 21, C_{34} \approx 15\end{array}$ \\
\hline $26,32-26,44$ & 26,40 & Integer & 82 & $\begin{array}{c}C_{01} \approx 1, C_{02} \approx 5, C_{03} \approx 13, C_{04} \approx 32, C_{12}=4, \\
C_{13}=12, C_{23} \approx 8, C_{14}=31, C_{24}=27, C_{34}=19\end{array}$ \\
\hline $28,52-28,60$ & 28,50 & $\begin{array}{l}\text { Integer } \pi- \\
\text { shifted }\end{array}$ & 78 & $\begin{array}{l}C_{01} \approx 1, C_{02} \approx 6, C_{03} \approx 14, C_{04} \approx 35, C_{12} \approx 4, \\
C_{13} \approx 13, C_{23} \approx 9, C_{14}=33, C_{24}=29, C_{34} \approx 21\end{array}$ \\
\hline
\end{tabular}


By observing the results of the tables can be affirmed that when the contrast is optimum the coefficient values of the non-paraxial equation take exact integer values. It means that several propagating waves arriving in phase contribute to self-image formation at the corresponding distance. Besides, for images with optimum contrast the coefficients are related by $C_{m n}=C_{q n}-C_{q m}$, where $q, m, n$ are integers and $0 \leq$ $\mathrm{q}<m<n$ where as usual $m$ and $n$ represent the order number of allowed propagating waves. In particular, the relation $C_{12}=C_{q 2}-C_{q 1}$ holds for gratings with periods between $2 \lambda$ and $4,2 \lambda$. On the other hand, just few propagating waves contribute to self image formation in the lower contrast cases, where is observed that for several $C_{m n}$ approximate their values to an integer number.

\section{Conclusions}

On the basis of the results shown, it can be concluded that the non-paraxial Talbot effect in free space and at optical frequencies for gratings of high density and period between $2 \lambda$ and $4,2 \lambda$ lacks of spatial axial periodicity in the self-images location. We note further that the formation of self-images on this scale in free space is achieved by the superposition of propagating waves without the participation of evanescent waves. Besides, when these self-images having good contrast can be observed the vast majority of the propagating waves interfere in phase at the positions where are formed resembling thereby the paraxial case. Also, others which are called quasi-self-images and have acceptable contrast are formed where only few propagating waves contribute in phase, which makes of this case different from the classical phenomenon combined with the non-axial periodicity location. Finally, according to the results reported in Table 1 for the 1,623 $\lambda$ period grating, its self-image locations have an approximate axial periodicity and agree with the Rayleigh formula. In addition, half period replicas appear as in the paraxial case where the period grating is much larger than the wavelength.

\section{Acknowledgments}

E. Arrieta. thanks Centro de Investigaciones Ópticas research stay which enabled him to carry out this work. Part of this work was presented at IX Iberoamerican Meeting on Optics and XII Iberoamerican Meeting on Optics, Lasers and Applications (RIAO / OPTILAS) 2016. 